\title{
Precisely Translating Computed Tomography Diagnosis Accuracy into Therapeutic Intervention by a Carbon-lodine Conjugated Polymer
}

\section{Mingming Yin}

Huazhong University of Science and Technology

\section{Xiaoming Liu}

Wuhan Union Hospital

\section{Ziqiao Lei}

Huazhong University of Science and Technology

\section{Yuting Gao}

China University of Geosciences

Jiacheng Liu

Wuhan Union Hospital

\section{Sidan Tian}

Huazhong University of Science and Technology

\section{Zhiwen Liang}

Huazhong University of Science and Technology

\section{Ye Wang}

Huazhong University of Science and Technology

\section{Fanling Meng}

Huazhong University of Science and Technology

Liang Luo ( $\sim$ liangluo@hust.edu.cn )

Huazhong University of Science and Technology https://orcid.org/0000-0001-9274-1866

\section{Article}

Keywords: CT imaging, contrast agents, poly(diiododiacetylene), fiducial markers, image-guided radiotherapy

Posted Date: February 16th, 2022

DOI: https://doi.org/10.21203/rs.3.rs-1332069/v1

License: (c) (1) This work is licensed under a Creative Commons Attribution 4.0 International License. Read Full License 
Version of Record: A version of this preprint was published at Nature Communications on May 12th, 2022. See the published version at https://doi.org/10.1038/s41467-022-30263-1. 


\section{Abstract}

The boomed development of precision medicine has underlined an immense need for ultraefficient, multifunctional markers to bridge diagnostic and therapeutic procedures. Particularly, ultraefficient X-ray computed tomography (CT) contrast agents that can precisely translate diagnostic findings into optimal therapeutic intervention represents an unsurmountable clinical challenge. Here, poly(diiododiacetylene) (PIDA), a conjugated polymer composed of only carbon and iodine atoms, is reported as an ultraefficient CT contrast agent to bridge CT diagnostic imaging with therapeutic intervention. PIDA has an unusually high iodine payload ( $>84 \mathrm{wt} \%$ ), and the aggregation of nanofibrous PIDA can further amplify CT intensity for over 10 folds with improved geometrical and positional stability in vivo. Moreover, with a highly conjugated backbone, PIDA is in deep blue color, making it dually visible by both CT imaging and naked eyes. The supreme performance of PIDA in CT-guided preoperative planning and visualization-guided surgery has been validated on orthotopic xenograft rat models. More strikingly, PIDA excels clinical fiducial markers of imaging-guided radiotherapy in efficiency and biocompatibility, and exhibits successful guidance of robotic radiotherapy on Beagles, demonstrating great clinical potential to translate CT diagnosis accuracy into therapeutic intervention for precision medicine.

\section{Introduction}

X-ray computed tomography (CT) is one of the most powerful diagnosis and therapy guidance techniques, with a leading frequency of use and hospital availablity ${ }^{1-4}$. Contrast agents with high X-ray attenuation, which typically contain high atomic number elements such as iodine, barium, and gold, are required to increase CT sensitivity and to better visualize the tissue of interest ${ }^{5-8}$, especially when identifying solid malignancies with small sizes and at early stages ${ }^{9-11}$. However, current clinically available CT contrast agents, majorly based on iodinated small compounds, suffer from rapid clearance and insufficient effectiveness-only large doses at molar concentrations or tens of grams can provide adequate $\mathrm{CT}$ imaging contrast ${ }^{12-14}$. In addition, while modern multimodal imaging techniques can identify tumor lesions at increasingly small sizes, the intraoperative localization and precise excision of these nonvisible and nonpalpable lesions become very difficult. Isotope-based radio-guided

localization ${ }^{15-17}$ and colored/fluorescent dyes ${ }^{18-22}$ have been developed to improve surgical guidance. However, these technologies are still afflicted with fast diffusion and short half-life of the marker materials. Moreover, most lesion positioning agents for instruments (e.g. CT imaging) cannot be readily visualized by surgeons in the operation procedures.

An ideal CT contrast agent that can bridge diagnostic imaging and therapeutic interventions should maximize the absolute CT attenuation difference between the tissue of interest and the surroundings by minimal doses, and maintain imaging properties throughout the course of preoperative CT scan and the designated therapies, which usually takes several hours to one day. More favorably, the contrast agent should be visible across CT imaging and naked eyes to improve the outcomes of surgical guidance. On the other hand, such contrast agents are highly desired in image-guided radiation therapy (IGRT), an 
emerging technology that has been introduced to improve the precision and lower the treatmentassociated toxicities of radiotherapy ${ }^{23-25}$. IGRT essentially relies on the accurate positioning of lesions throughout the radiotherapy 26,27 . However, tumors rarely show a fixed location during irradiation or treatment due to respiratory movement, changes in organ filling, etc ${ }^{28-30}$. Radiopaque fiducial markers were typically implanted into the tumor tissue for tumor tracking and radiation beam alignment. Au-based fiducial markers are most commonly used in clinic, but they often lead to severe side effects, including inflammation, swollen, bleeding, and consequently position shift ${ }^{31-33}$. Their permanent in vivo stay is also a threat in prognosis and management. In addition, Au-based fiducial markers generate streak artifacts in CT images 34,35 , therefore hindering the visibility of surrounding tissues and affecting the accuracy of dose distribution calculation and treatment planning ${ }^{36,37}$. CT contrast agents that can overcome these deficiencies with excellent biocompatibility and maximized performance are in urgent need to advance the technology of IGRT.

Polydiacetylenes have recently emerged as interesting, biocompatible functional materials for biomedical applications ${ }^{38-41}$. We and others have demonstrated the synthesis of a variety of functionalized polydiacetylenes through a host-guest co-crystal strategy ${ }^{42-45}$. Considering the wide clinical utility of iodine-containing aromatic compounds for CT imaging, we hypothesize that poly(diiododiacetylene), or PIDA (Fig. 1a), represents an exceptional solution to the general problems of CT contrast agents, attributed to its extremely high payload of iodine and many other intriguing characteristics. PIDA contains a polydiacetylene backbone with only iodine atom substituents, and its iodine mass content can reach as high as $84.1 \%$. Moreover, given its nanofibrous nature, PIDA should easily aggregate and accumulate on the target tissue for prolonged retention. In addition, the highly planar conjugated backbone of PIDA endows the material a deep blue color, so that it is easily distinguishable from the surrounding tissues by naked eyes, making it ideal for CT-guided preoperative planning and visualization-guided intraoperative target positioning. We are herein inspired to explore in this study the suitability of applying PIDA as a contrast agent for CT imaging, surgical guidance, and IGRT, in view of its ultrahigh X-ray attenuation efficiency, dual visibility by CT and naked eyes, and excellent in vivo retention.

\section{Results}

\section{Preparation of PIDA and ultrahigh X-ray attenuation}

The formation of PIDA requires the topochemical polymerization, i.e., the head-to-tail 1,4-polymerization, of diiodobutadiyne monomers assembled in an appropriate arrangement (Fig. 1a). To achieve the required spacial alignment of monomers for this topochemical polymerization, we use host-guest supramolecular scaffolds based on halogen bonds, the Lewis acid-base interactions between the monomer and functionalized Lewis bases ${ }^{44,46}$. In previous attempts to prepare PIDA ${ }^{42,43,47}$, biscyanoalkyl or bispyridyl oxalamides were employed as hosts to align monomer guests in co-crystals. However, they could not yield PIDA with refined structure in large quantities ${ }^{43}$, or only generated cocrystals that required high pressure to initiate the polymerization ${ }^{48}$. Here, using $N^{1}, N^{2}$-bis(2-(pyridin-3- 
yl)ethyl)oxalamide (1, structure shown in Supplementary Fig. 1) as the host ${ }^{49}$, we are able to produce PIDA co-crystals with well-defined structure (Fig. 1b) on gram-scale, so that the possibility of further application of PIDA is boosted. PIDA 1 co-crystals exhibit a metallic appearance that is characteristic of a high degree of polymerization (Fig. 1b, bottom right), and scanning electron microscope (SEM) image reveals their very smooth surface, with one dimension (010) much smaller than the other two dimensions (Fig. 1b, top right).

In order to isolate PIDA from the co-crystals and formulate it in aqueous media for in vivo applications, we utilized an amphiphilic polymer, polyethylene glycol-grafted poly(maleic anhydride-alt-1-octadecene), or C18-PMH-PEG, as the surfactant ${ }^{50}$. Sonication followed by dialysis of a mixture of the PIDA• 1 cocrystals and C18-PMH-PEG in water resulted in a stable blue aqueous suspension (Fig. 1C). The UV-visible absorption spectrum of the blue PIDA suspension (Fig. 1d) exhibited a maximal absorption peak at 652 $\mathrm{nm}$ with a broad shoulder at $750 \mathrm{~nm}$, attributed to the planar backbone of the material. The Raman spectrum of the suspension was consistent with that of the co-crystals (Fig. 1e), indicating that the structure of PIDA was preserved during the process. Transmission electron microscope (TEM) imaging confirmed that the blue suspension mainly contained well-dispersed nanofibers with diameters of 30-50 $\mathrm{nm}$ (Fig. 1f). Moreover, the elemental analysis of the nanofibers by an energy-dispersive X-ray (EDX) detector showed the existence of only carbon $(15.7 \mathrm{wt} \%)$ and iodine $(84.3 \mathrm{wt} \%)$ atoms, which was consistent with the theoretical value of PIDA (15.9 wt\% for carbon and $84.1 \mathrm{wt} \%$ for iodine) and evidenced the thorough removal of host 1 (Fig. 1g).

Effective payload of iodine atoms is crucial to the high performance of CT contrast agents ${ }^{12}$. However, current clinically available CT contrast agents all have iodine mass contents of less than $50 \%$, such as iohexol (46.4 wt\%), iopromide (48.1 wt\%), and iodixanol (49.1 wt\%). The $84.1 \mathrm{wt} \%$ iodine content of PIDA warranted it an ultrahigh X-ray attenuation ability. The CT value, an indicator in terms of Hounsfield unit $(\mathrm{HU})$ that measures the ability of a material to attenuate X-rays, of the PIDA suspension increased linearly with the iodine concentration (Fig. 1h), indicating that PIDA was well dispersed in the media. The slope of $\mathrm{HU}$ values against sample concentrations for PIDA was 1.8 times higher than that for iohexol, suggesting a superior X-ray attenuation ability of PIDA. More strikingly, when a PIDA suspension was condensed to form PIDA aggregates (Supplementary Fig. 2), its CT value dramatically increased over 10 times, from $213 \mathrm{HU}$ to $2475 \mathrm{HU}$ (Fig. 1i). To validate this aggregation-induced CT intensity amplification, we injected a PIDA suspension and an iohexol solution (both with an iodine concentration of $4 \mathrm{mg} \mathrm{mL}^{-1}$ ) into two porcine tissues, respectively (Supplementary Fig. 3). The contrast ratio of PIDA in the pork muscle tissue $(87.8 \%)$ was 4 times higher than that of iohexol $(21.7 \%)$, whereas in the fat tissue, the contrast ratio of PIDA (251.7\%) was 17 times higher than that of iohexol (14.5\%). In addition, PIDA nanofibers were observed to accumulate in the injection sites, with a significantly longer in situ retention time than iohexol.

\section{PIDA-based ultraefficient CT imaging in rats}


Inspired by the remarkable X-ray attenuation ability of PIDA, we injected PIDA suspensions ([I]: $4 \mathrm{mg}$ $\mathrm{mL}^{-1}$ ) into rat legs to evaluate their performance in CT imaging. lohexol solutions ([I]: $4 \mathrm{mg} \mathrm{mL}^{-1}$ ) were injected in the control rat. Ideally, a contrast agent should improve the absolute CT attenuation of the target tissue to more than twice of the surrounding tissue and fluids ${ }^{12}$. The background CT signal of rat muscle tissue was about $50 \mathrm{HU}$, and the CT value of intramuscularly injected PIDA could reach $260 \mathrm{HU}$ at 2 min after injection (Fig. 2a). As a comparison, the CT value of iohexol was around $150 \mathrm{HU}$ at that time (Fig. 2b). Markedly, the CT value of PIDA continued to increase as time progressed and reached $660 \mathrm{HU}$ at $2 \mathrm{~h}$ after injection, possibly attributed to the aggregation of PIDA at the injection site. The PIDA converged at the injection site and formed a well-defined marker with fixed geometry and position. Its CT value maintained at a high level of above $500 \mathrm{HU}$ for over $6 \mathrm{~h}$ (Fig. 2d and Supplementary Fig. 4), a typical period required for CT-guided preoperative planning, surgery scheduling, and surgical procedure in hospital. As a comparison, the injected iohexol was barely visible at 2 min after injection, and its CT value dropped promptly to less than $100 \mathrm{HU}$ within $1 \mathrm{~h}$ (Fig. 2b, 2d and Supplementary Fig. 5). Since an effective diagnostic dose of iohexol was typically in molar concentrations, we next applied a high concentration iohexol solution ([l]: $100 \mathrm{mg} \mathrm{mL}^{-1}$ ) in rats. Although the initially formed marker had a high CT value $(1700 \mathrm{HU})$, it decayed very fast and the CT value decreased dramatically down to $100 \mathrm{HU}$ at $2 \mathrm{~h}$ post injection (Fig. 2c, 2d and Supplementary Fig. 6), suggesting its rapid diffusion and clearance. The PIDA marker was also clearly visible under regular X-ray examination, which further confirmed the ultraefficient X-ray attenuation of PIDA (Supplementary Fig. 7). The great performance of PIDA in rat CT imaging hence demonstrates its great potential to meet clinical marker needs, given its strong CT intensity at ultralow iodine concentrations, prolonged tissue retention time, as well as high geometrical and positional stability.

\section{CT and naked eyes dual-visible surgical marker}

The practicability of current imaging markers for surgical guidance is seriously restricted in that they cannot accurately translate diagnostic image findings into specific therapeutic intervention. A surgical marker that can bridge this gap by identifying occult lesions both under instrumental guidance and by direct visual observation is highly desirable in clinic ${ }^{18,51}$. In addition to the ultraefficient CT imaging characteristic, PIDA can preserve its unique deep blue color in vivo for various time after the injection (Supplementary Fig. 8), hence enabling precise localization of the targeting lesions by both CT imaging and naked eyes. To evaluate if PIDA can serve as such a dual-visible surgical marker, we injected PIDA suspensions on the tumor periphery of an orthotopic xenograft rat model under CT imaging guidance (Fig. 3a), and examined the assistance of PIDA labeling in identifying tumor resection margin (Fig. 3b). As expected, the injected PIDA markers maintained high level CT values with fixed geometry and positions on the tumor periphery for a period of $6 \mathrm{~h}$ (Fig. 3c and Supplementary Fig. 9). When we dissected the rats at $24 \mathrm{~h}$ after injection, the blue colored PIDA markers were still clearly visible by naked eyes and distributed around the nonpalpable tumor lesion to indicate the surgical margin of the targeted tumor tissue (Fig. 3d). As a comparison, the CT signals of iohexol control (at the same iodine dose) disappeared quickly (Supplementary Fig. 10). more strikingly, after we intratumorally injected the PIDA 
suspension, the PIDA nanofibers diffused and fulfilled the whole tumor tissue in $2 \mathrm{~h}$ (Fig. 3e), clearly demonstrating the margin line between the tumor tissue and the normal tissue. The complete tumor infiltration of PDDA nanofibers had been confirmed by magnetic resonance (MR) imaging (Fig. $3 \mathrm{~g}$ ) and CT imaging (Fig. 3h), which could be directly used to identify the tumor boundary by naked eyes in the surgery (Fig. 3f, 3i). Intraoperative identification of tumor boundary remains a great challenge in clinic, and it is especially difficult for small and nonpalpable tumor lesions. The dual-visible PIDA allowed precise intraoperative localization of occult orthotopic tumors, demonstrating a great potential to directly translate diagnosis accuracy to therapeutic intervention for enhanced clinical surgical outcomes.

\section{Fiducial marker for stereotactic body radiation therapy}

In addition to the surgical marker, PIDA can also serve as an excellent candidate for fiducial makers in stereotactic body radiation therapy (SBRT). SBRT is an advanced approach of IGRT to precision radiotherapy, which employs robotic radiotherapy (RRT) to deliver precise doses of radiation with extreme accuracy (Fig. 4a) ${ }^{52,53}$. Because of its high precision, SBRT has to take real-time tumor movement into account, while tumor motions during the respiratory cycle is a major contributor to targeting uncertainty. RRT utilizes an orthogonal X-ray imaging system and an optical respiratory tracking system to build a real-time correlation model between the tumor and the external abdominal surface of the patient (Supplementary Fig. 11). The established correlation model then allows the RRT to estimate tumor position based on optical tracking, which is sent to the robotic positioning system to enable real-time adjustment of the tumor position during respiratory cycles. In clinic, Au-based fiducial markers are most commonly implanted in tumor tissues to serve as a surrogate for tumor positioning in clinical practice. However, they are prone to cause local edema, inflammation, and positioning shifts ${ }^{31-33,52}$, therefore impeding patients from receiving desired clinical benefits. PIDA, on the other hand, represents a straightforward solution to the problems associated with Au-based fiducial markers.

To make a direct comparison with the commercially available Au marker, PIDA was casted into a similar shape and implanted into the liver of a rat through puncture needles under the guidance of CT imaging (Fig. 4b). On Day 7 post implantation, MR imaging revealed severe local edema caused by the implanted Au marker (Fig. 4c), which was confirmed by the optical image of the dissected tissue (yellow arrows, Fig. 4d). As a comparison, no PIDA-caused edema was observed either on MR image (Fig. 4e) or dissected tissue (Fig. 4f). The blood analysis, the hematoxylin and eosin (H\&E), and TdT-mediated dUTP nick end labeling (TUNEL) stain of the tissue around the implanted markers also indicated excellent biocompatibility of PIDA as the implantable marker (Supplementary Table 1 and Supplementary Fig. 12).

Moreover, the implanted Au marker contacted loosely to the surrounding tissues (Fig. 4d), which implied a high probability of position shift or even off target during clinical application. As a comparison, PIDA marker attached tightly to the surrounding tissues (Fig. 4f), suggesting a stable positioning in SBRT.

To further assess the positioning accuracy of PIDA as a fiducial marker in SBRT, we implanted a PIDA maker into the liver of a Beagle, on which a clinical SBRT was executed afterwards (Fig. 4g). A vacuum cushion was used to fix the posture of the Beagle during the SBRT treatment and to ensure the 
consistency with the preoperative CT modeling (Fig. 4g, bottom inset). From CT imaging, the PIDA marker exhibited a strong CT signal in the liver of the Beagle (Fig. 4g, top inset), on basis of which a threedimensional distribution map of planned X-ray irradiation was generated for RRT on the Beagle (Fig. 4h). The real-time position of the PIDA marker in RRT was monitored by live X-ray imaging (green circle in Fig. $4 \mathrm{i}$ ), which moved close to the original position (yellow diamond in Fig. 4i, determined from the preoperative CT imaging) periodically during the respiratory cycle of the Beagle (Supplementary Video 1). In addition, the correlation error for RRT was measured based on the difference between the actual position of the target (green circle in Fig. 4i) and the predicted position of the target computed from the Beagle's respiratory movement (Fig. 4j). The average correlation error of the PIDA marker was $1.07 \pm 0.55$ $\mathrm{mm}$ (Fig. 4k), lower than that of Au markers typically found in clinic $(1.7 \pm 1.1 \mathrm{~mm})^{54}$, indicating a good agreement with the correlation models for the execution of the planned RRT (Fig. 4h). The uncertainty of the PIDA marker, which provided the detection uncertainty value for the fiducial extraction algorithm, was $9.10 \pm 2.30 \%$ (Fig. 4I), far below the uncertainty threshold parameter of $40 \%{ }^{55}$. PIDA hence exhibited an exceptional positioning accuracy in SBRT of the Beagle.

An effective SBRT replies not only on the accurate positioning of fiducial markers in RRT, but also on the precise radiation dose calculation, which can be easily affected by artifacts in CT images. Compared with the nontrivial metal artifacts that were seen in the CT images of the rat implanted with an Au fiducial marker (Fig. 5a), PIDA presented a much more efficient labeling effect with minimal artifact interference (Fig. 5b). To further quantify the advantage of PIDA fiducial marker in artifact reduction, we used a thorax phantom with movable plugs to simulate the CT imaging of a whole human body in SBRT (Supplementary Fig. 13). Each movable plug in the phantom simulated a specific human organ, and inserting PIDA or Au marker to a plug mimicked the labeling of the corresponding organ. The CT imaging showed that PIDA could mark the target organ much more effectively than Au, given its negligible artifacts (Fig. 5c). The quantified CT intensities on the circled spots distributing near the PIDA marker (Fig. 5d, 5e) were almost identical to those of the unmarked sample (Control), suggesting that the artifactfree PIDA marker showed no influence on the visibility of the surrounding tissues. As a comparison, the Au marker exhibited a significant decrease of CT intensity in the tangential direction of the plug, as well as a considerable increase of CT intensity in the perpendicular normal direction. In addition, the calculated radiation dose distribution for RRT planning based on the Au marker apparently deviated from the actual dose distribution in the Control group (Fig. $5 f, 5 g$ ). As a comparison, the PIDA marker resulted in a more accurate dose distribution calculation (Fig. $5 \mathrm{~g}$ ), showing a significant advantage over Au-based fiducial marker in assisting the treatment planning system for the precise radiation delivery in SBRT.

\section{Biocompatibility and Biodegradability of PIDA}

To further ensure the safety of PIDA for future applications as a CT contrast agent, we comprehensively characterized its biocompatibility and biodegradability. We first mixed different concentrations of PIDA suspensions with rat red blood cells (RBCs). Triton was used as a positive control and set as $100 \%$ hemolysis. The hemolysis rates of all PIDA suspensions were below $5 \%$, suggesting that PIDA did not cause hemolysis at tested concentrations. In addition, we incubated PIDA suspensions at different 
concentrations with 4T1 cells (mouse breast cancer cells), NIH 3T3 cells (mouse embryonic fibroblasts), and HEK 293T (human embryonic kidney cells) for $12 \mathrm{~h}$ (Fig. 6b). The cell viabilities of all PIDAcontaining groups, tested by MTT assays, maintained above $90 \%$, showing the good biocompatibility of PIDA.

Moreover, the weight of the experimental rats increased normally over time (Fig. 6c), and no significant difference in blood analysis and liver/kidney function tests between PIDA-injected rats and normal rats was observed (Supplementary Fig. 14), indicating that PIDA did not affect the physiological status of the animals. In addition, we have showed that the CT value of injected PIDA suspension ([I]: $4 \mathrm{mg} \mathrm{mL}^{-1}$ ) could maintain over $500 \mathrm{HU}$ for $6 \mathrm{~h}$ (Fig. 2d). In fact, it still remained high at around $400 \mathrm{HU}$ for $24 \mathrm{~h}$ (Fig. $6 \mathrm{~d}, 6 \mathrm{f})$, which should guarantee the completion of most CT-involved operations in hospital. The injected PIDA became invisible by CT imaging on Day 7 post injection (Fig. 6e), and on Day 21, the CT value decreased to a level close to iohexol ([l]: $4 \mathrm{mg} \mathrm{mL}^{-1}$ ), which was injected into another rat synchronously with PIDA (Fig. 6f). The CT value and the volume of the PIDA fiducial marker in rat liver also decreased over time (Supplementary Fig. 15). The gradual degradation and bioabsorbability of PIDA can be explained by the fact that polydiacetylene backbone is cleavable by reactive oxygen species ${ }^{56}$, and the relatively labile carbon-iodine bond can also facilitate the process ${ }^{57}$. In addition, no obvious damage was found in major organs at 1 day or 1 week after the injection (Supplementary Fig. 16), and the H\&E stains of main organ tissues also confirmed that PIDA did not cause any toxicity during the whole process (Fig. $6 \mathrm{~g}$ ). The excellent biosafety and biodegradability of PIDA therefore pave the way for translational studies of PIDA-based CT contrast agents.

\section{Discussion}

The tremendous development of CT imaging has revolutionized its ability to accurately diagnose malignancies as well as to promote surgical interventions for improved clinical benefits. However, current clinically available contrast agents are less than optimal. Most iodinated contrast agents have rapid renal clearance and insufficient effectiveness, while metal-based contrast agents are hampered by strong artifacts and biosafety issues. In this work, we demonstrate for the first time that how well PIDA, a conjugated polymer of carbon and iodine, can address these deficiencies and serve as an ideal contrast agent for CT imaging and related therapy intervention. With an unusually high iodine payload of $84.1 \%$, the intrinsic X-ray attenuation ability of PIDA has surpassed most iodine-based contrast agents. Moreover, PIDA exhibits a striking aggregation-induced amplification in CT intensity attributed to its nanofibrous nature, leading to an over 10 -fold boost in local CT intensity and significantly enhanced tissue retention as well as geometrical and positional stability. In addition, PIDA has a deep blue color because of its highly conjugated backbone, so that it can be dually visible by both CT imaging and naked eyes, making it a perfect marker candidate for CT-guided preoperative planning and intraoperative localization of nonpalpable tumor lesions. More notably, compared with metal-based fiducial markers, PIDA excels on its biocompatibility, fixed positioning, and minimal artifacts while maintaining an 
effectively high CT intensity. These characteristics are critical for the precise calculation of dose distribution in treatment planning systems, as well as for the accurate radiation delivery in robotic SBRT.

Biomedical applications and clinical translations of conjugated molecular materials have been long pursued. The successful demonstration of the above advantages of PIDA on rat and Beagle models foresees its great translational potential for clinical applications, not only expanding anatomical localization labeling which current technologies may be difficult to apply, but also encouraging the explorations of conjugated molecular materials to solve challenging clinical problems. The clinical translation of PIDA contrast agents, together with formulating PIDA for targeted CT imaging, are currently on the way.

\section{Declarations}

\section{Acknowledgements}

We thank Yuncheng Cai for the help on crystal structure analysis, and Ming Yang for the help on animal experiments. This work is supported by the National Natural Science Foundation of China (21877042), the National Basic Research Plan of China (2018YFA0208903), and Huazhong University Startup Fund. We thank the Analytical and Testing Center of Huazhong University of Science and Technology for related analysis.

\section{Author contributions}

M. Y., X. L. and Z. L. contributed equally to this work. M. Y., X. L. and Z. L. designed and performed the experiments. Y.G., S.T., Z.L. and Y. W. provided helpful suggestions to this work. J. L. participated in some of the animal work. F. M. and L. L. conceived and obtained funding for the project, oversaw the research and wrote the paper. All authors discussed the results and commented on the paper.

\section{Competing interests}

The authors declare no competing interests.

\section{References}

1. Lell, M.M. \& Kachelriess, M. Recent and upcoming technological developments in computed tomography: high speed, low dose, deep learning, multienergy. Invest. Radiol. 55, 8-19 (2020).

2. Leipsic, J. et al. SCCT guidelines for the interpretation and reporting of coronary CT angiography: a report of the Society of Cardiovascular Computed Tomography Guidelines Committee. J. Cardiovasc. Comput. Tomogr. 8, 342-358 (2014).

3. Udugama, B. et al. Diagnosing COVID-19: the disease and tools for detection. ACS Nano 14, 38223835 (2020). 
4. Dai, W.C. et al. CT imaging and differential diagnosis of COVID-19. J. Can. Assoc. Radiol. 71, 195200 (2020).

5. Lee, N., Choi, S.H. \& Hyeon, T. Nano-sized CT contrast agents. Adv. Mater. 25, 2641-2660 (2013).

6. Freilich, J.M. et al. Lipiodol as a fiducial marker for image-guided radiation therapy for bladder cancer. Int. Braz. J. Urol. 40, 190-197 (2014).

7. Liu, Y.L. et al. Hybrid BaYbF 5 nanoparticles: novel binary contrast agent for high-resolution in vivo Xray computed tomography angiography. Adv. Healthcare Mater. 1, 461 (2012).

8. Liu, Y. et al. A high-performance ytterbium-based nanoparticulate contrast agent for in vivo X-ray computed tomography imaging. Angew. Chem., Int. Ed. 51, 1437-1442 (2012).

9. Fass, L. Imaging and cancer: a review. Mol. Oncol. 2, 115-152 (2008).

10. Liu, Y., Ai, K. \& Lu, L. Nanoparticulate X-ray computed tomography contrast agents: from design validation to in vivo applications. Acc. Chem. Res. 45, 1817-1827 (2012).

11. Kinsella, J.M. et al. X-ray computed tomography imaging of breast cancer by using targeted peptidelabeled bismuth sulfide nanoparticles. Angew. Chem., Int. Ed. 50, 12308 (2011).

12. Lusic, H. \& Grinstaff, M.W. X-ray-computed tomography contrast agents. Chem. Rev. 113, 1641-1666 (2013).

13. Torchilin, V.P., Frank-Kamenetsky Md Fau - Wolf, G.L. \& Wolf, G.L. CT visualization of blood pool in rats by using long-circulating, iodine-containing micelles. Academic Radiology 6, 61-65 (1999).

14. Davenport, M.S. et al. Contrast material-induced nephrotoxicity and intravenous low-osmolality iodinated contrast material. Radiology 267, 94-105(2013).

15. Valdes Olmos, R.A. et al. Advances in radioguided surgery in oncology. Q. J. Nucl. Med. Mol. Imaging 61, 247-270 (2017).

16. Schaarup-Jensen, H. et al. Injectable iodine-125 labeled tissue marker for radioactive localization of non-palpable breast lesions. Acta Biomater. 65, 197-202 (2018).

17. Norregaard, K. et al. ${ }^{18} \mathrm{~F}$-FDG PET/CT-based early treatment response evaluation of nanoparticleassisted photothermal cancer therapy. PLoS One 12, e0177997 (2017).

18. Mehendale, V.G., Chaudhari, N.C., Shenoy, S.N. \& Mehendale, A.V. Henna as a durable preoperative skin marker. World J. Surg. 35, 311-315 (2011).

19. Chan, B.K., Wiseberg-Firtell, J.A., Jois, R.H., Jensen, K. \& Audisio, R.A. Localization techniques for guided surgical excision of non-palpable breast lesions. Cochrane Database Syst. Rev. CD009206 (2015).

20. Gioux, S., Choi, H.S. \& Frangioni, J.V. Image-guided surgery using invisible near-infrared light: fundamentals of clinical translation. Mol. Imaging 9, 237-255 (2010).

21. Hu, Z. et al. First-in-human liver-tumour surgery guided by multispectral fluorescence imaging in the visible and near-infrared-I/Il windows. Nat. Biomed. Eng. 4, 259-271 (2020).

22. Zhu, S., Tian, R., Antaris, A.L., Chen, X. \& Dai, H. Near-infrared-II molecular dyes for cancer imaging and surgery. Adv. Mater. 31, 1900321 (2019). 
23. Jaffray, D.A. Image-guided radiotherapy: from current concept to future perspectives. Nat. Rev. Clin. Oncol. 9, 688-699 (2012).

24. Zelefsky, M.J. et al. Improved clinical outcomes with high-dose image guided radiotherapy compared with non-IGRT for the treatment of clinically localized prostate cancer. Int. J. Radiat. Oncol. Biol. Phys. 84, 125-129 (2012).

25. Alexander, S.E., Kinsella, J., McNair, H.A. \& Tree, A.C. National survey of fiducial marker insertion for prostate image guided radiotherapy. Radiography $24,275-282$ (2018).

26. Gill, S. et al. Patient-reported complications from fiducial marker implantation for prostate imageguided radiotherapy. Br. J. Radiol. 85, 1011-1017 (2012).

27. Brown, K.H., Ghita, M., Schettino, G., Prise, K.M. \& Butterworth, K.T. Evaluation of a novel liquid fiducial marker, BioXmark ${ }^{\circledR}$, for small animal image-guided radiotherapy applications. Cancers 12 , 1276 (2020).

28. Jiang, S.B. Radiotherapy of mobile tumors. Semin. Radiat. Oncol. 16, 239-248 (2006).

29. Kirilova, A. et al. Three-dimensional motion of liver tumors using cine-magnetic resonance imaging. Int. J. Radiat. Oncol. Biol. Phys. 71, 1189-1195 (2008).

30. Jackson, W.C. et al. Stereotactic body radiation therapy for localized prostate cancer: a systematic review and meta-analysis of over 6,000 patients treated on prospective studies. Int. J. Radiat. Oncol. Biol. Phys. 104, 778-789 (2019).

31. Oldrini, G. et al. Implantation of fiducial markers in the liver for stereotactic body radiation therapy: feasibility and results. Diagn. Interv. Imaging 96, 589-592 (2015).

32. Bhagat, N. et al. Complications associated with the percutaneous insertion of fiducial markers in the thorax. Cardiovasc Intervent Radiol 33, 1186-1191 (2010).

33. Delouya, G., Carrier, J.F., Beliveau-Nadeau, D., Donath, D. \& Taussky, D. Migration of intraprostatic fiducial markers and its influence on the matching quality in external beam radiation therapy for prostate cancer. Radiother. Oncol. 96, 43-47 (2010).

34. Slagowski, J.M. et al. Evaluation of the visibility and artifacts of 11 common fiducial markers for image guided stereotactic body radiation therapy in the abdomen. Pract. Radiat. Oncol. 10, 434-442 (2020).

35. Liang, Z. et al. Artificial intelligence-based framework in evaluating intrafraction motion for liver cancer robotic stereotactic body radiation therapy with fiducial tracking. Med. Phys. 47, 5482-5489 (2020).

36. Wolterink, J.M., Leiner, T., Viergever, M.A. \& Isgum, I. Generative adversarial networks for noise reduction in low-dose CT. IEEE Trans. Med. Imaging 36, 2536-2545 (2017).

37. Zhang, Y. et al. Efficient CT metal artifact reduction based on fractional-order curvature diffusion. Comput. Math. Methods Med. 2011, 173748 (2011).

38. Sun, X., Chen, T., Huang, S., Li, L. \& Peng, H. Chromatic polydiacetylene with novel sensitivity. Chem. Soc. Rev. 39, 4244-4257 (2010). 
39. Chen, X., Zhou, G., Peng, X. \& Yoon, J. Biosensors and chemosensors based on the optical responses of polydiacetylenes. Chem. Soc. Rev. 41, 4610-4630 (2012).

40. Fang, F., Meng, F. \& Luo, L. Recent advances on polydiacetylene-based smart materials for biomedical applications. Mater. Chem. Front. 4, 1089-1104 (2020).

41. Tian, S. et al. Polydiacetylene-based ultrastrong bioorthogonal Raman probes for targeted live-cell Raman imaging. Nat. Commun. 11, 81 (2020).

42. Sun, A., Lauher, J.W. \& Goroff, N.S. Preparation of poly(diiododiacetylene), an ordered conjugated polymer of carbon and iodine. Science 312, 1030 (2006).

43. Luo, L. et al. Poly(diiododiacetylene): preparation, isolation, and full characterization of a very simple poly(diacetylene). J. Am. Chem. Soc. 130, 7702-7709 (2008).

44. DeCicco, R.C., Luo, L. \& Goroff, N.S. Exploiting unsaturated carbon-iodine compounds for the preparation of carbon-rich materials. Acc. Chem. Res. 52, 2080-2089 (2019).

45. Jin, H., Young, C.N., Halada, G.P., Phillips, B.L. \& Goroff, N.S. Synthesis of the stable ordered conjugated polymer poly(dibromodiacetylene) from an explosive monomer. Angew. Chem., Int. Ed. 54, 14690-14695 (2015).

46. Metrangolo, P., Neukirch, H., Pilati, T. \& Resnati, G. Halogen bonding based recognition processes: a world parallel to hydrogen bonding. Acc. Chem. Res 38, 386-395 (2005).

47. Lauher, J.W., Fowler, F.W. \& Goroff, N.S. Single-crystal-to-single-crystal topochemical polymerizations by design. Acc. Chem. Res 41, 1215-1229 (2008).

48. Jin, H., Plonka, A.M., Parise, J.B. \& Goroff, N.S. Pressure induced topochemical polymerization of diiodobutadiyne: a single-crystal-to-single-crystal transformation. CrystEngComm 15, 3106-3110 (2013).

49. Wilhelm, C. 1,4 Poly(diiododiacetylene): production and characterization. Ph.D. dissertation, Stony Brook University (2009).

50. Liu, X. et al. Optimization of surface chemistry on single-walled carbon nanotubes for in vivo photothermal ablation of tumors. Biomaterials 32, 144-151 (2011).

51. Wang, W. et al. Carbohydrate based biomarkers enable hybrid near infrared fluorescence and ${ }^{64} \mathrm{Cu}$ based radio-guidance for improved surgical precision. Nanotheranostics 5, 448-460 (2021).

52. Scher, N. et al. Safety and efficacy of fiducial marker implantation for robotic stereotactic body radiation therapy with fiducial tracking. Radiat. Oncol. 14, 167 (2019).

53. Casutt, A. et al. Endobronchial coil spring fiducial markers for Cyberknife ${ }^{\circledR}$ stereotactic body radiation therapy. Respirology 26, 469-476 (2021).

54. Winter, J.D., Wong, R., Swaminath, A. \& Chow, T. Accuracy of robotic radiosurgical liver treatment throughout the respiratory cycle. Int. J. Radiat. Oncol. Biol. Phys. 93, 916-924 (2015).

55. Accuray Inc. Headquarters et al. Treatment delivery manual 10.5.x for CyberKnife ${ }^{\circledR}$ Robotic Radiosurgery System. Accuray Inc, Sunnyvale, CA, USA (2015). 
56. Tian, S. et al. Complete degradation of a conjugated polymer into green upcycling products by sunlight in air. J. Am. Chem. Soc. 143, 10054-10058 (2021).

57. Luo, L. et al. Room-temperature carbonization of poly(diiododiacetylene) by reaction with Lewis bases. J. Am. Chem. Soc. 133, 19274-19277 (2011).

\section{Supplementary Video}

Supplementary Video 1 is not available with this version

\section{Figures}

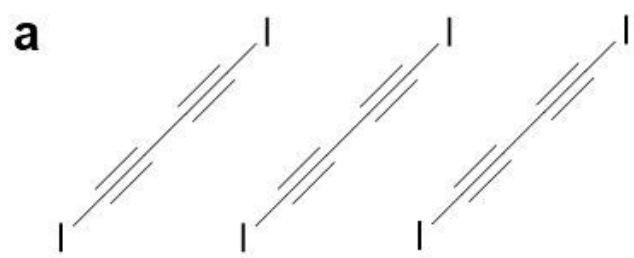

Diiodobutadiyne

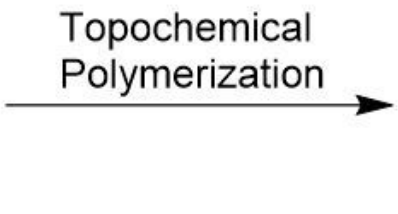

b

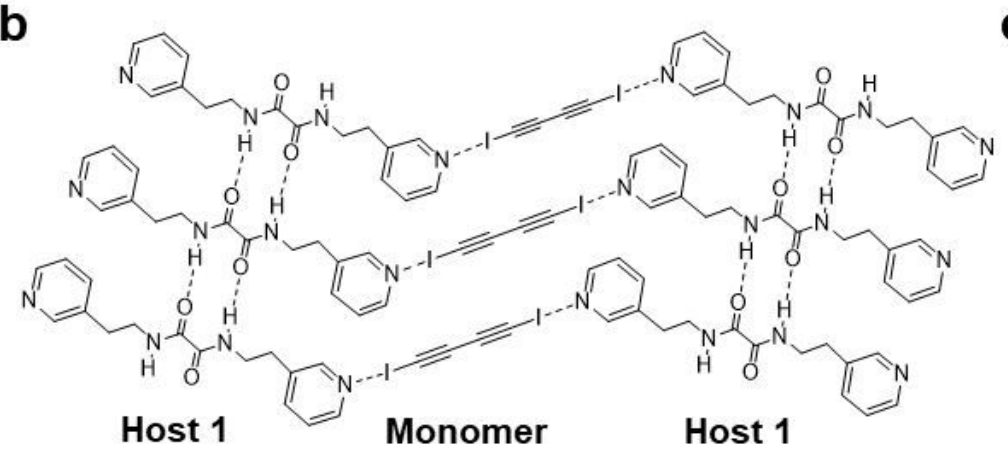

d

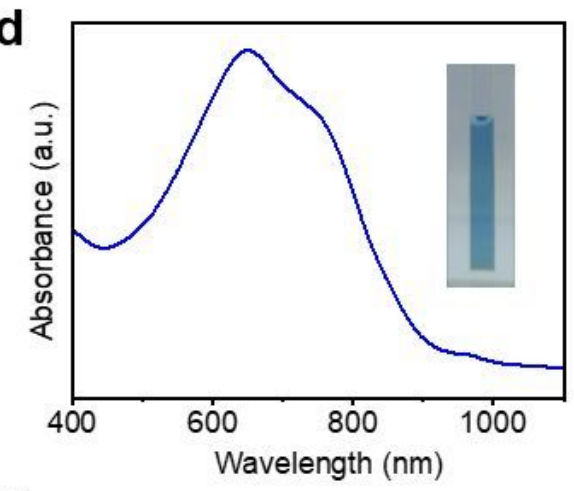

g

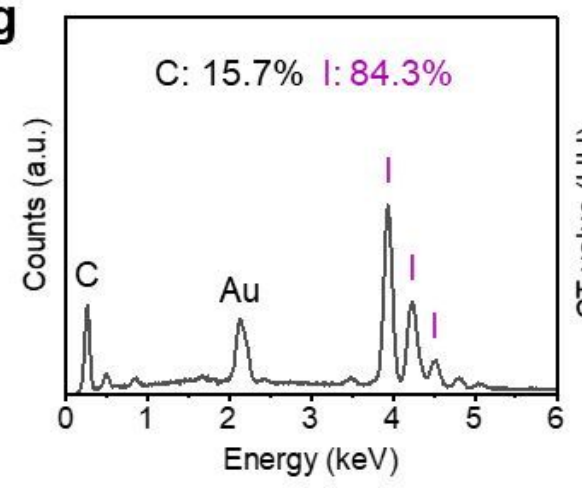

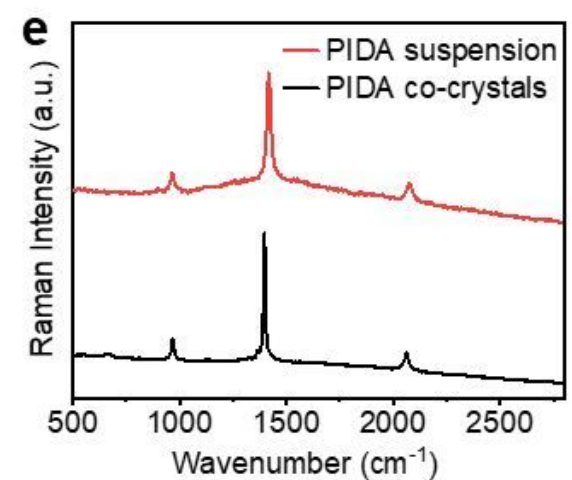

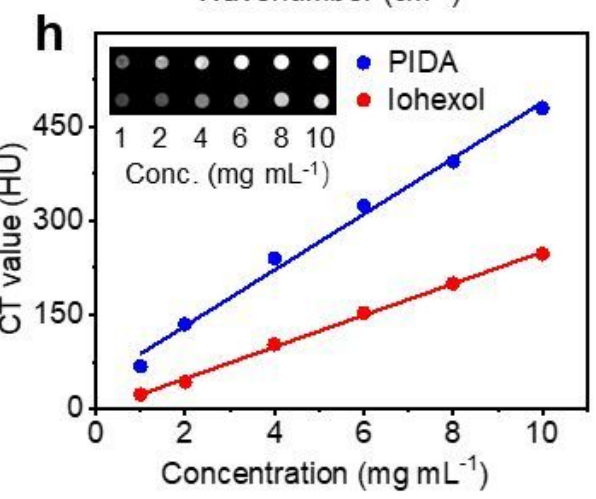

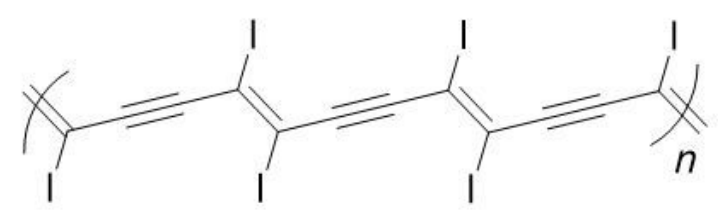

PIDA
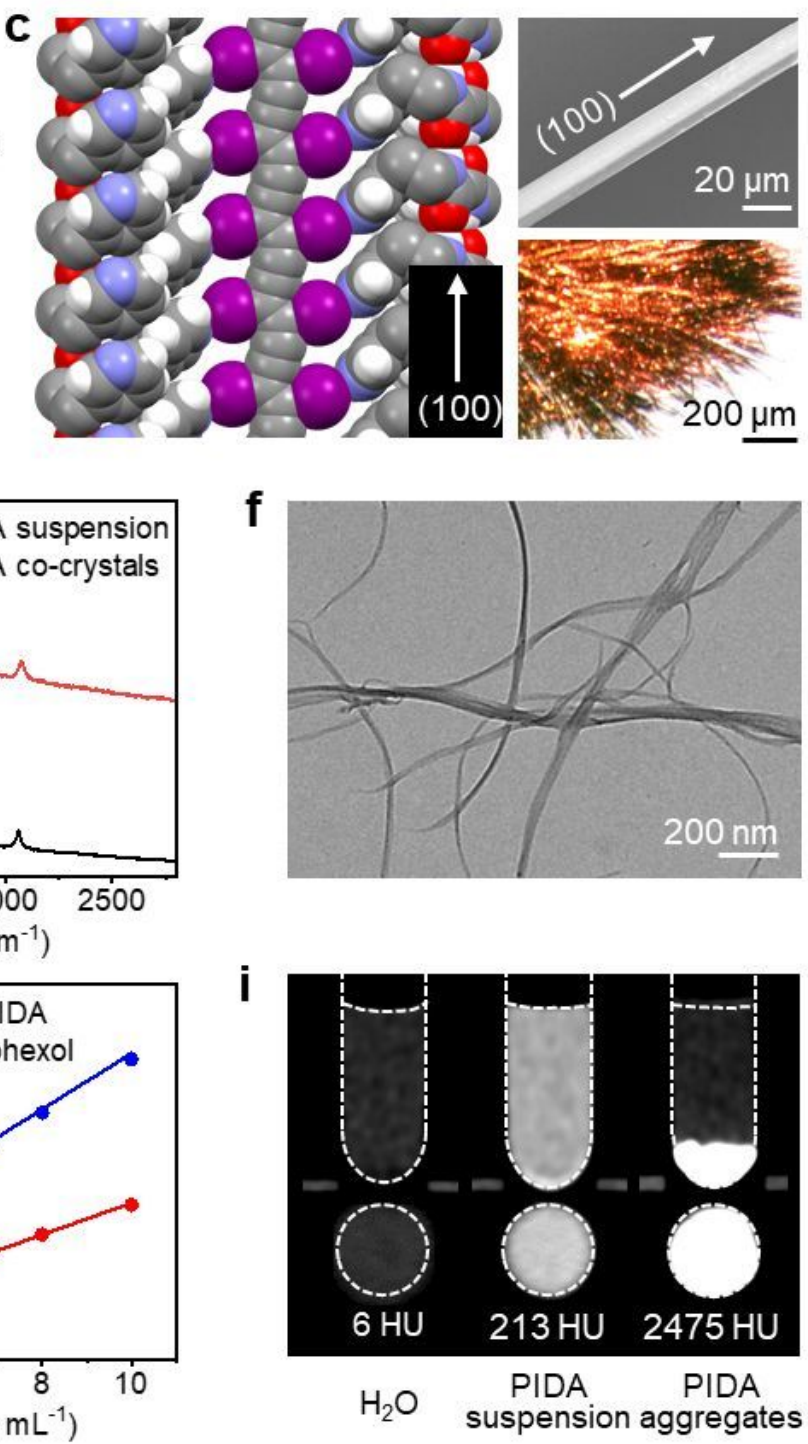


\section{Figure 1}

Preparation and characterization of PIDA. a Synthesis of PIDA by topochemical polymerization. $\mathbf{b}$ Hostguest supramolecular scaffold formed between Host 1 and monomer diiodobutadiyne. c Single-crystal structure of PIDA• 1 co-crystals determined by X-ray diffraction (left), and SEM (top right) and optical (bottom right) images of PIDA• 1 co-crystals. $\mathbf{d}$ Raman spectra of PIDA• 1 co-crystals and the prepared PIDA suspension. e UV-vis absorption spectrum of the PIDA suspension. Inset: Picture of the PIDA suspension. $\mathbf{f}$ TEM image of dispersed PIDA nanofibers in the PIDA suspension. $\mathbf{g}$ Elemental analysis of PIDA by EDX. $\mathbf{h}$ CT values of PIDA and iohexol as a function of sample concentrations. Inset: CT images of PIDA suspension and iohexol at corresponding concentrations. i CT images of pure water, PIDA suspension $\left(5 \mathrm{mg} \mathrm{mL}^{-1}\right)$, and PIDA aggregates. Top row: side view; Bottom row: Top view. 
a

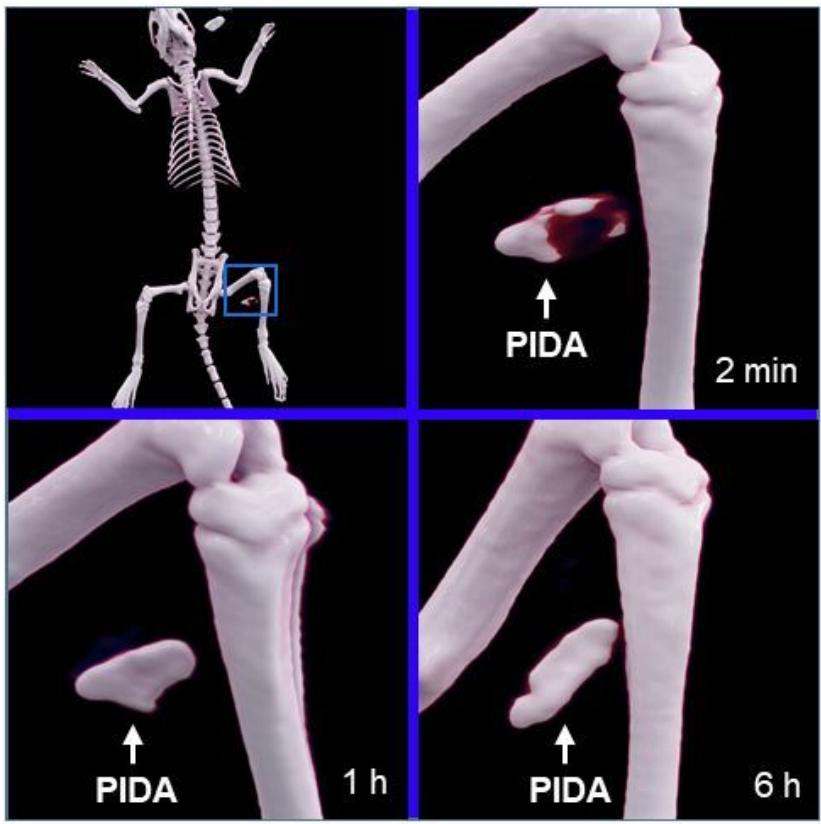

c

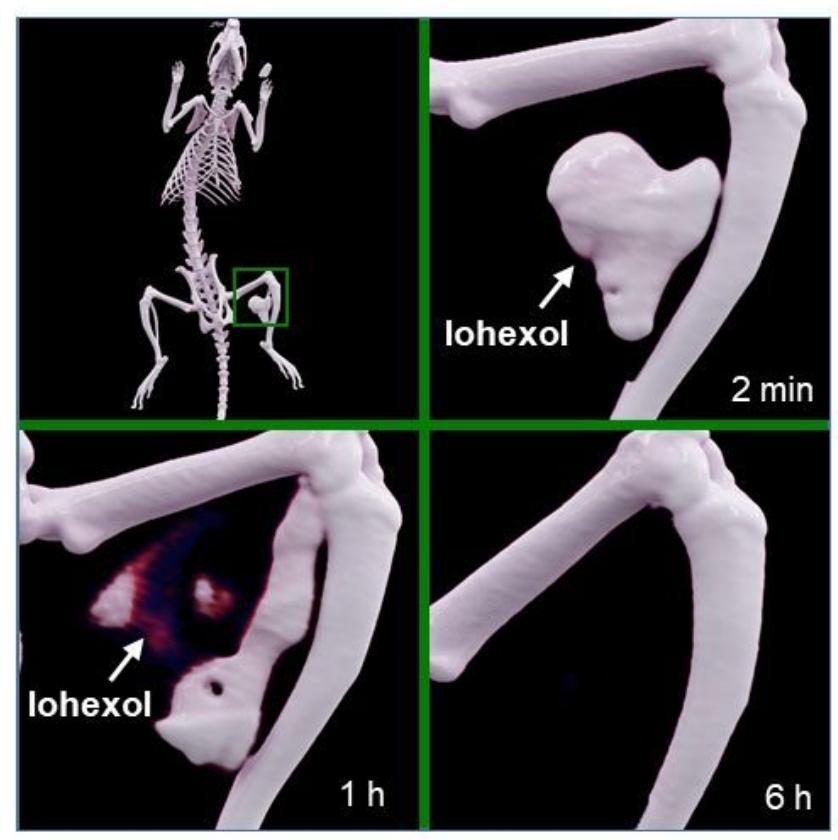

b

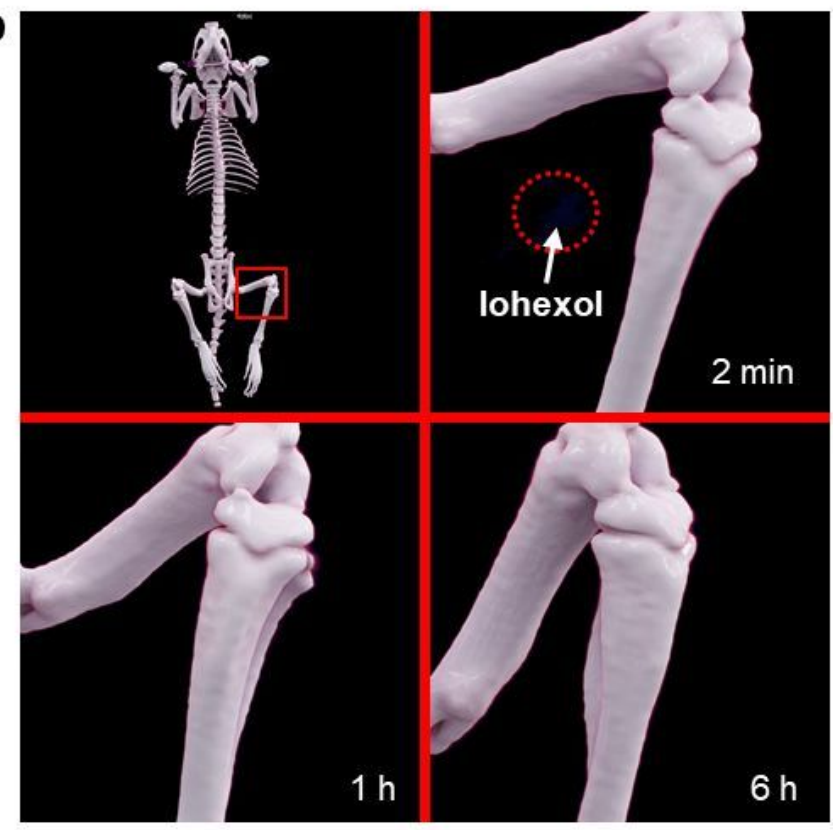

d

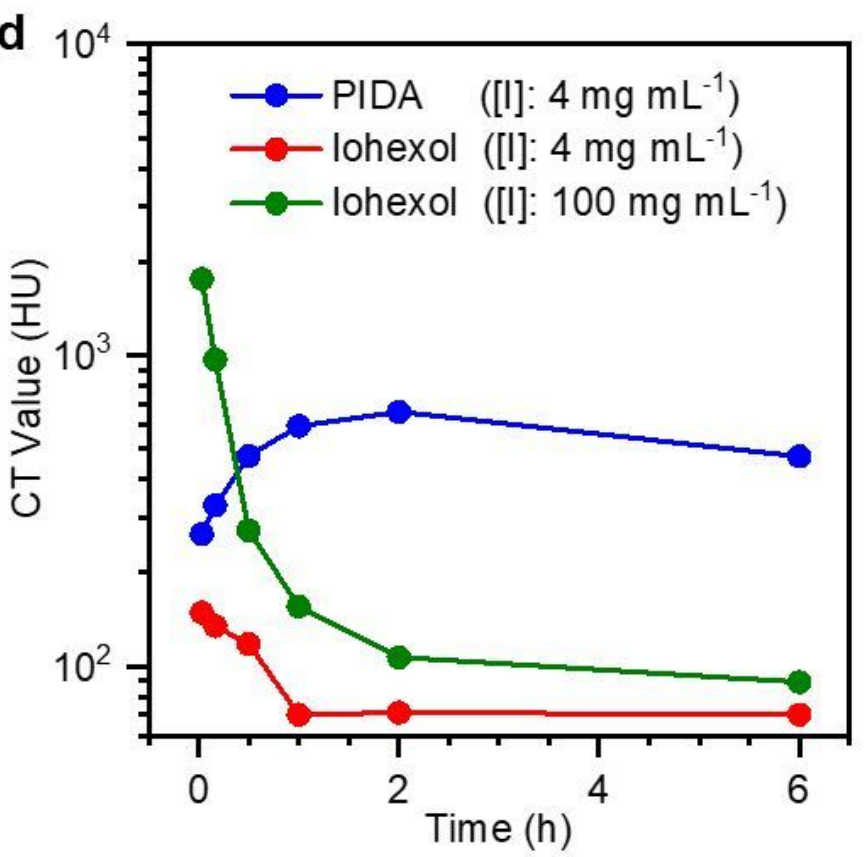

Figure 2

The performance of PIDA and iohexol in CT imaging of rats. a CT images of a rat at different time after being injected with $200 \mu \mathrm{L}$ PIDA suspension ([I]: $4 \mathrm{mg} \mathrm{mL}^{-1}$ ). b CT images of a rat at different time after being injected with $200 \mu \mathrm{L}$ of iohexol ([I]: $4 \mathrm{mg} \mathrm{mL}^{-1}$ ). $\mathrm{CCT}$ images of a rat at different time after being injected with $200 \mu \mathrm{L}$ of iohexol ([I]: $100 \mathrm{mg} \mathrm{mL}^{-1}$ ). $\mathbf{d}$ Plots of CT values of different markers in $\mathbf{a}$, $\mathbf{b}$, and $\mathbf{c}$ as a function of time post injection. 

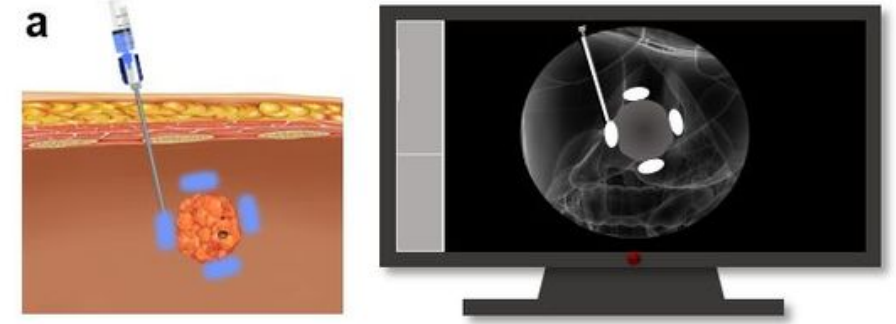

CT-guided tumor location b

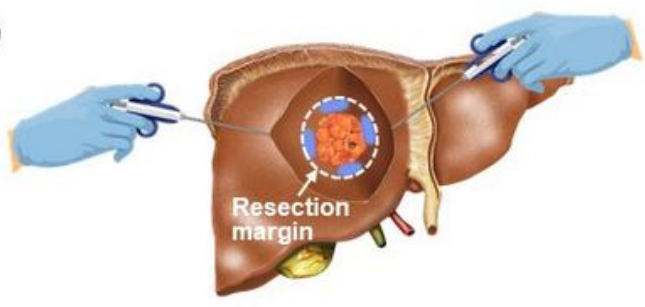

Marker-guided surgical resection
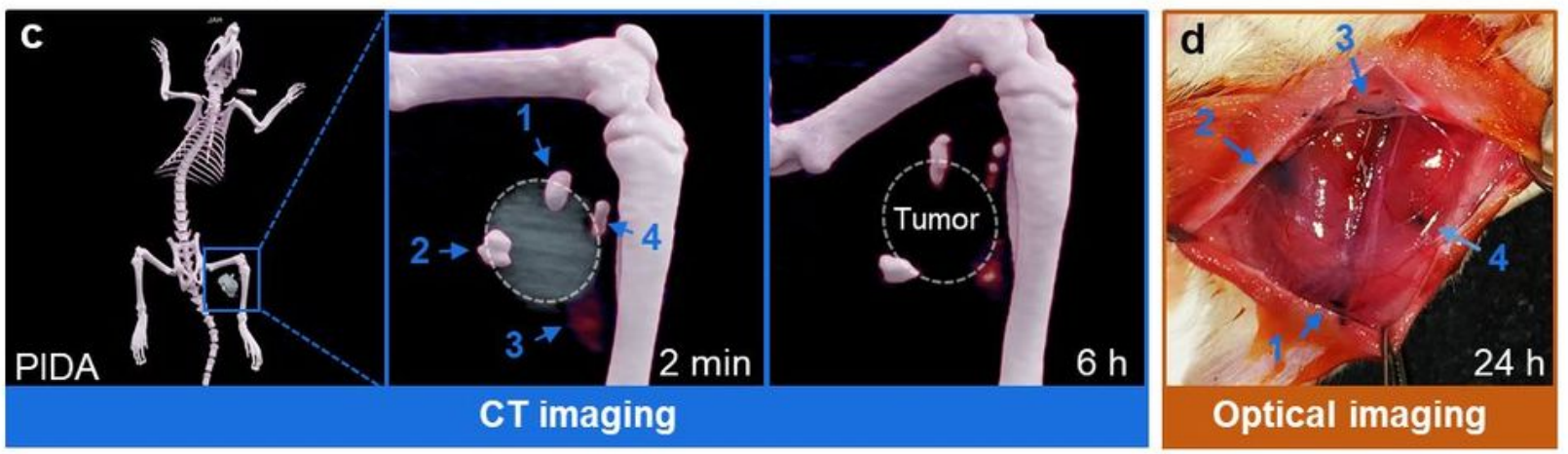

e

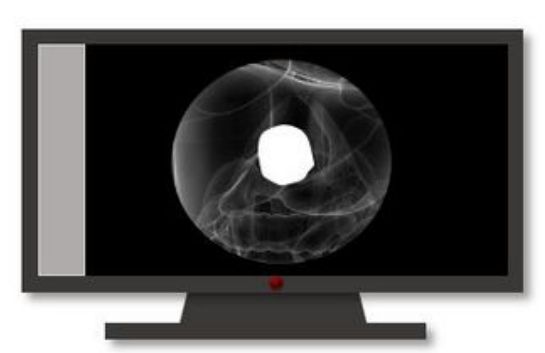

f

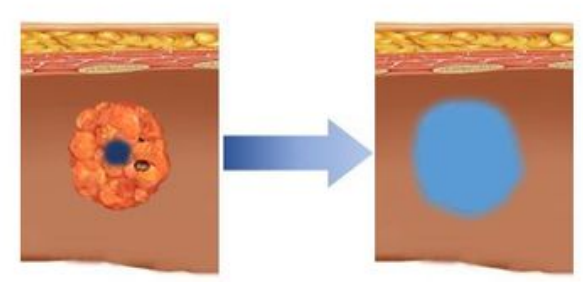

Tumor infiltration guided by $\mathrm{CT}$ imaging

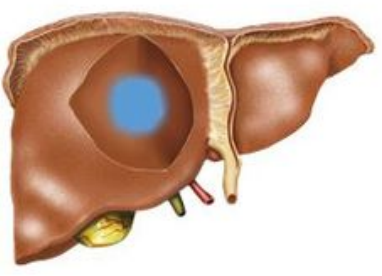

Surgical guidance

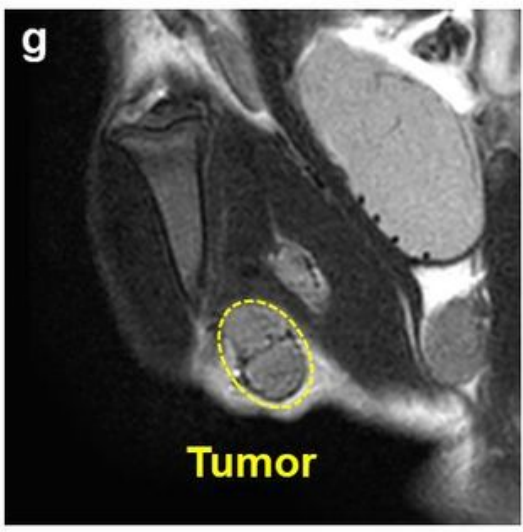

MR imaging

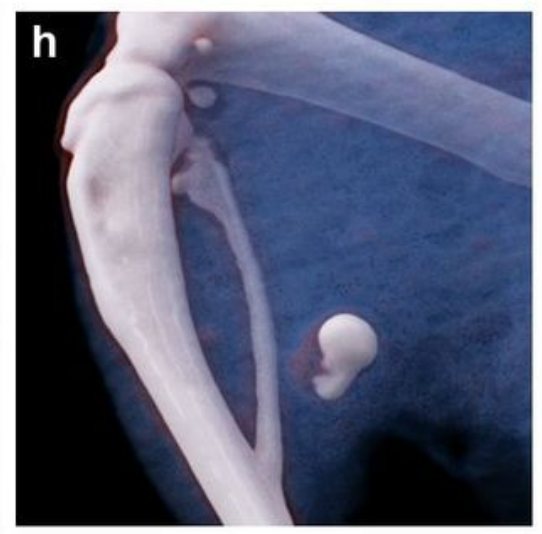

CT imaging

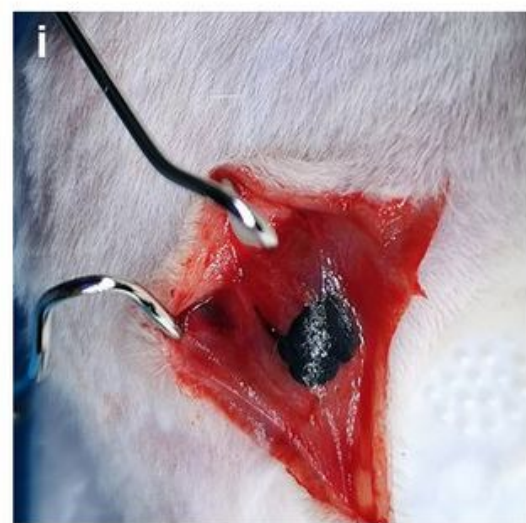

Optical imaging

Figure 3

CT-guided preoperative tumor labeling and visualization-guided surgery on basis of CT and eye dually visible PIDA. a, b Schematic illustration of tumor labeling by PIDA marker under CT guidance (a) and surgical resection of the labeled tumor guided by eye-visible blue PIDA (b). c CT images of a tumorbearing rat injected with a PIDA suspension at 4 spots on the tumor periphery ([I]: $4 \mathrm{mg} \mathrm{mL}^{-1}, 50 \mu \mathrm{L}$ each) over time. Tumors were omitted for better illustration for CT images of $6 \mathrm{~h}$. $\mathbf{d}$ Exposed orthotopic tumor 
sites of the rat injected with PIDA. 4 blue PIDA residues surrounding the tumor clearly determined the resection margin of the tumor. e Schematic illustration of CT-guided tumor infiltration of intratumorally injected PDDA suspension. $\mathbf{f}$ Tumor infiltration of PDDA nanofibers for tumor boundary determination. $\mathbf{g}$, h, i Complete tumor infiltration of PDDA nanofibers by MR imaging (g), CT imaging (h), and optical imaging (i).

a
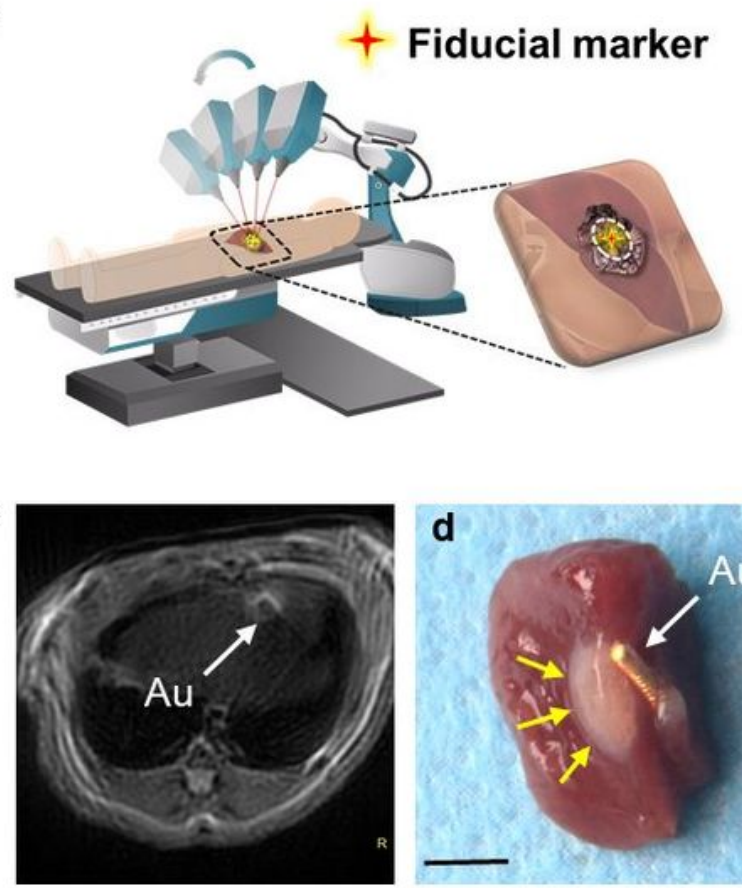

g

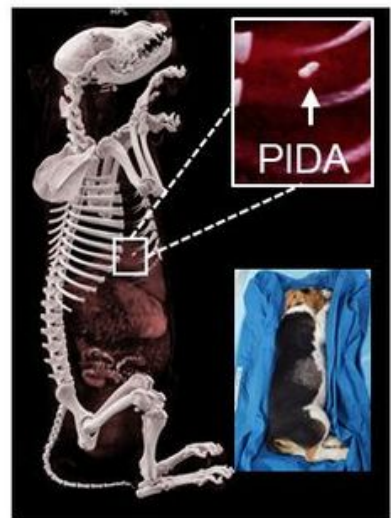

h
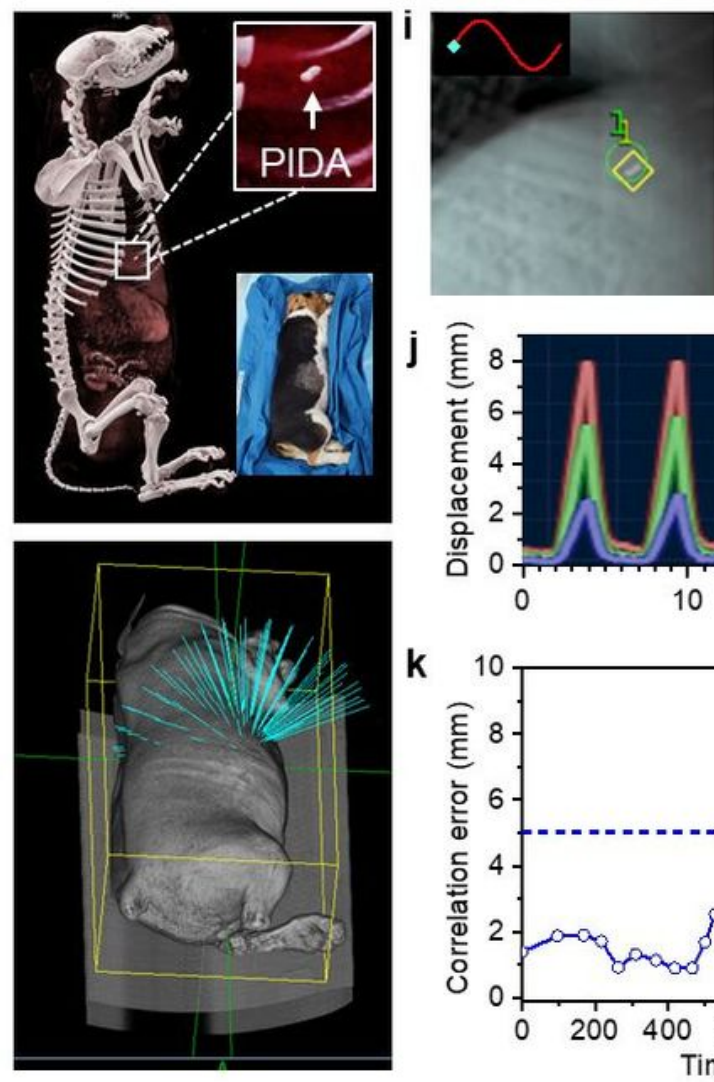

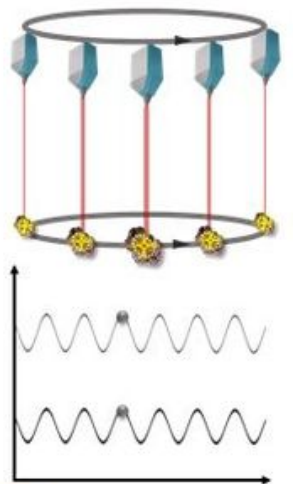

b
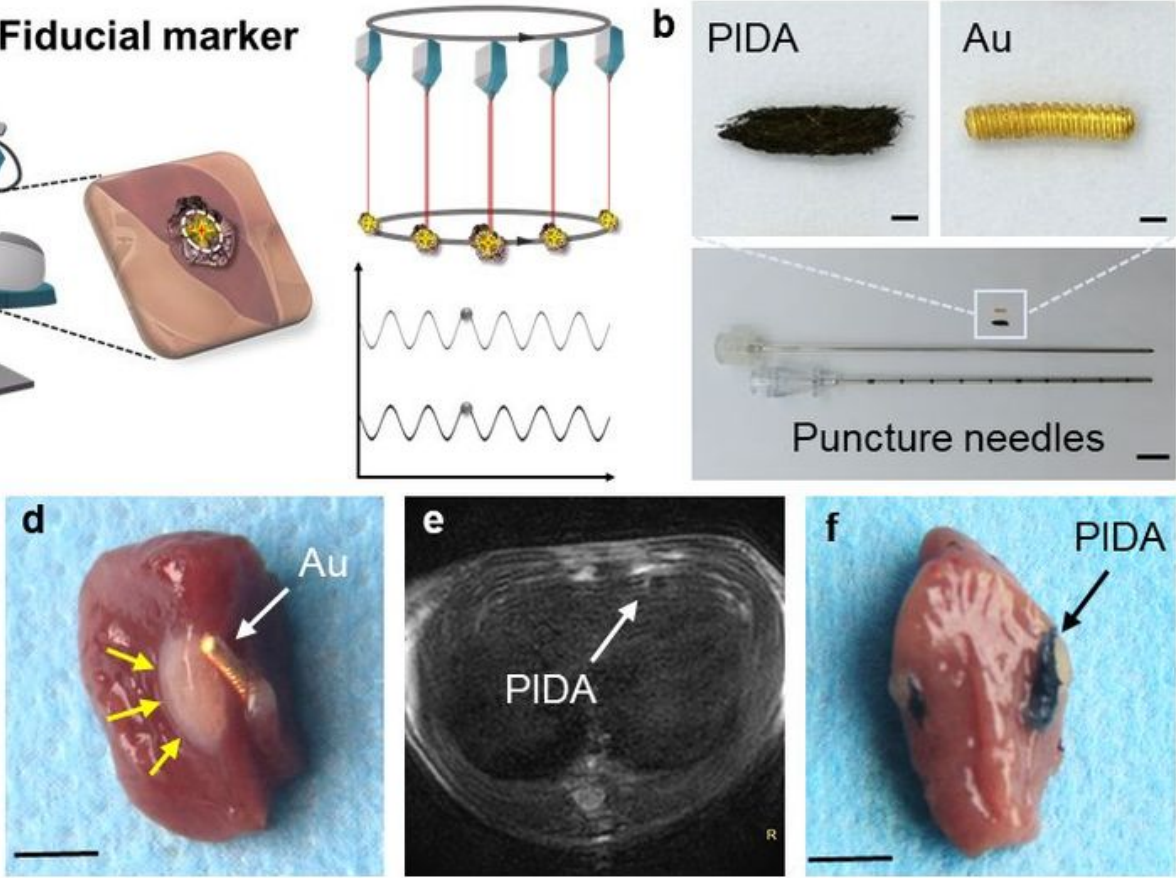

e
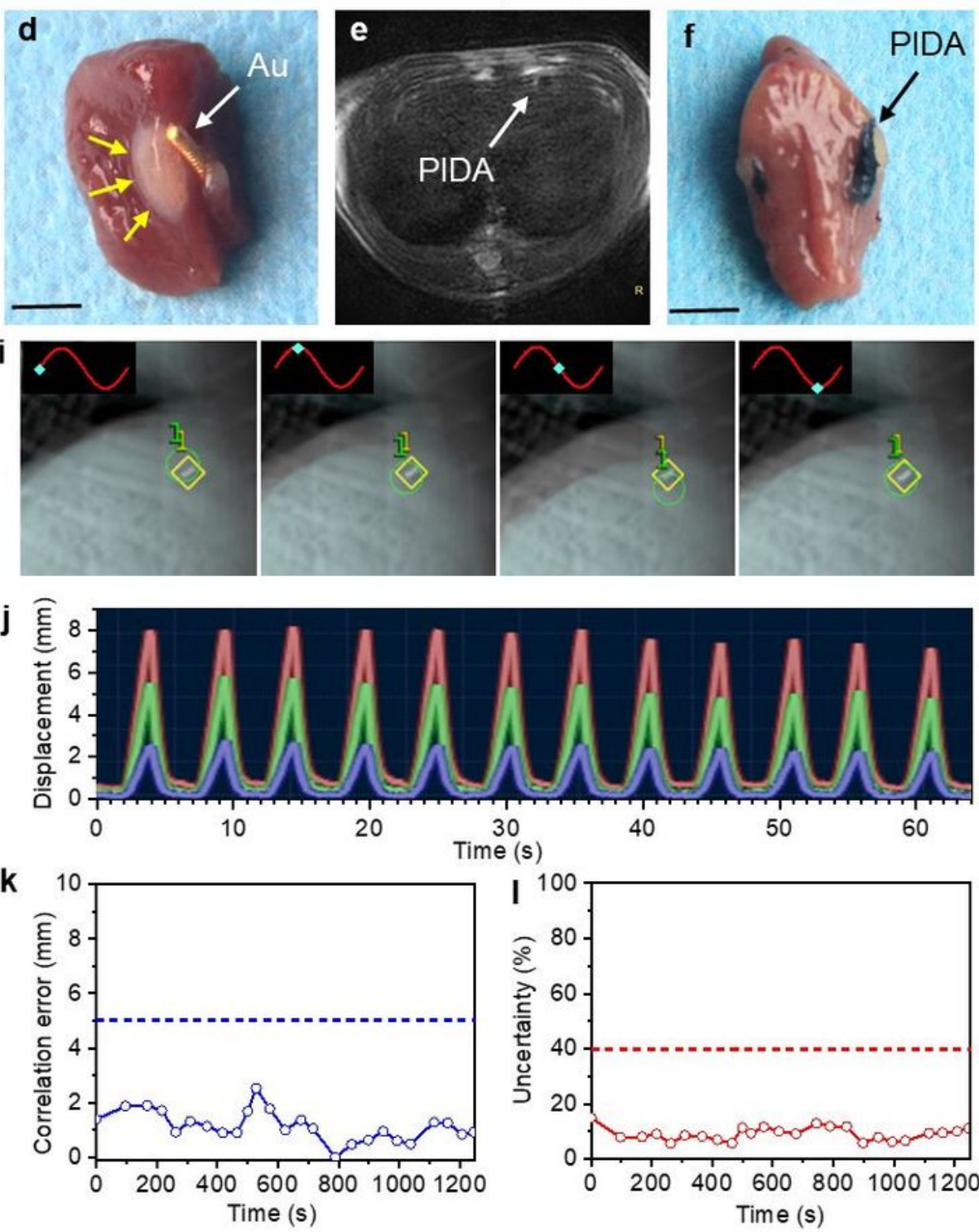

Figure 4 
The evaluation of PIDA as a fiducial maker in robotic SBRT. a Schematic diagram of robotic SBRT in a clinical setting. b Pictures of the puncture needles (Scale bars: $1 \mathrm{~cm}$ ), the PIDA marker (Scale bars: 1 $\mathrm{mm}$ ), and the Au marker used in clinic (Scale bars: $1 \mathrm{~mm}$ ). c MR image of a rat implanted with an Au marker. $\mathbf{d}$ Optical image of the resected rat liver tissue containing the Au maker, in which severe edema was observed (yellow arrows). Scale bar: $0.5 \mathrm{~cm}$. e MR image of a rat implanted with a PIDA marker. $\mathbf{f}$ Optical image of the resected rat liver tissue containing the PIDA maker. Scale bar: $0.5 \mathrm{~cm} . \mathbf{g}$ CT image of the Beagle implanted with a PIDA marker. White rectangle: PIDA in the Beagle liver; Bottom inset: The Beagle fixed in a memory cushion for SBRT. $\mathbf{h}$ Three-dimensional distribution map of planned X-ray radiation for RRT on the Beagle. i X-ray images of the Beagle liver at different phases during the respiratory cycle. Green circles: the positions of the PIDA maker on live X-ray images; Yellow diamonds: the original position of the PIDA marker on the $\mathrm{X}$-ray image simulated from preoperative CT modeling. $\mathbf{j}$ Beagle's respiratory movement during RRT determined by an optical respiratory tracking system. $\mathbf{k}$ Plot of the real-time correlation error during RRT. Blue dashed line indicates the threshold limit in clinic. I Plot of the real-time uncertainty during RRT. Red dashed line indicates the threshold limit in clinic. 
a
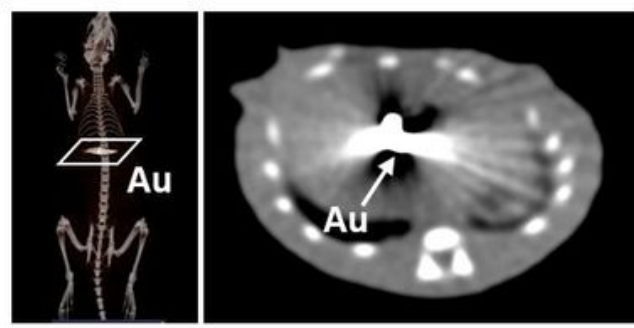

C
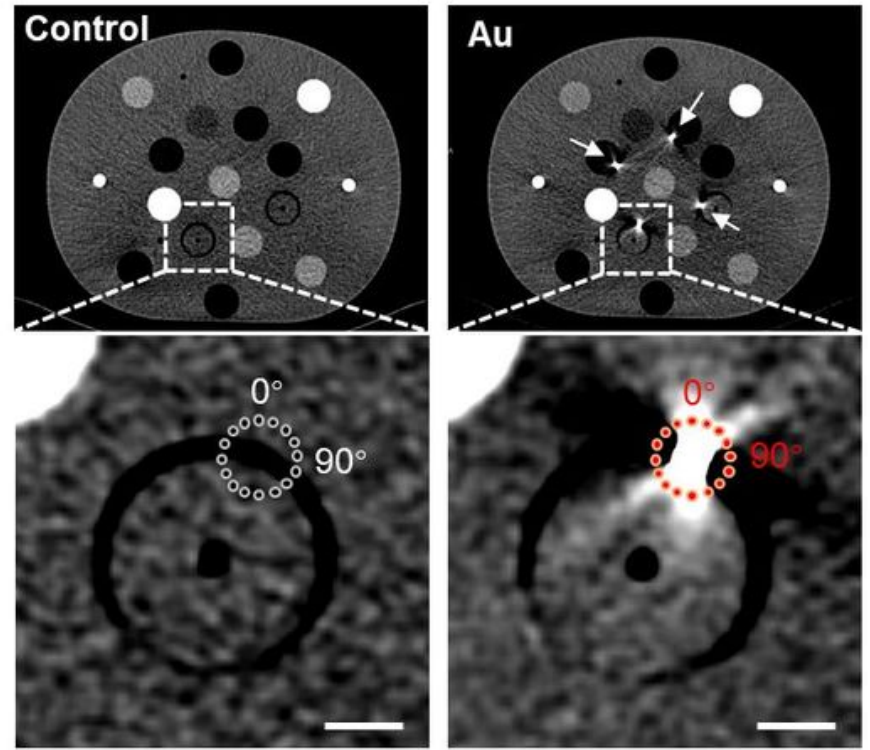

d
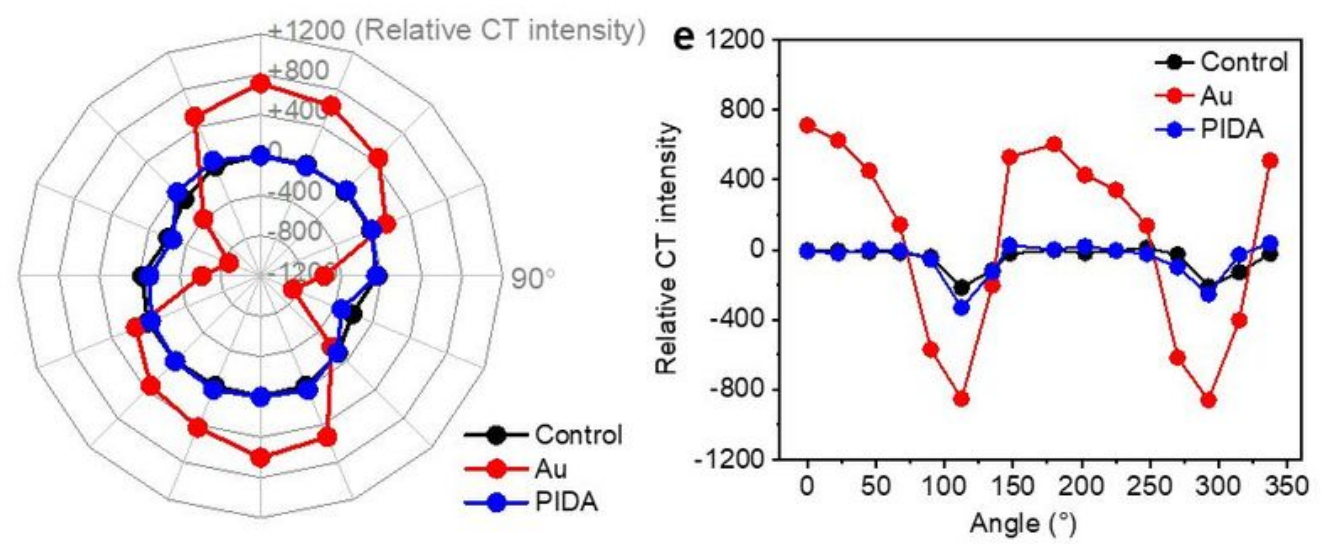

f

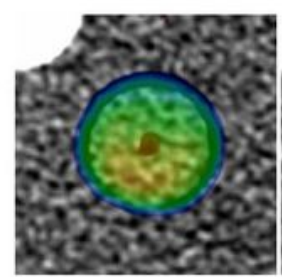

Control
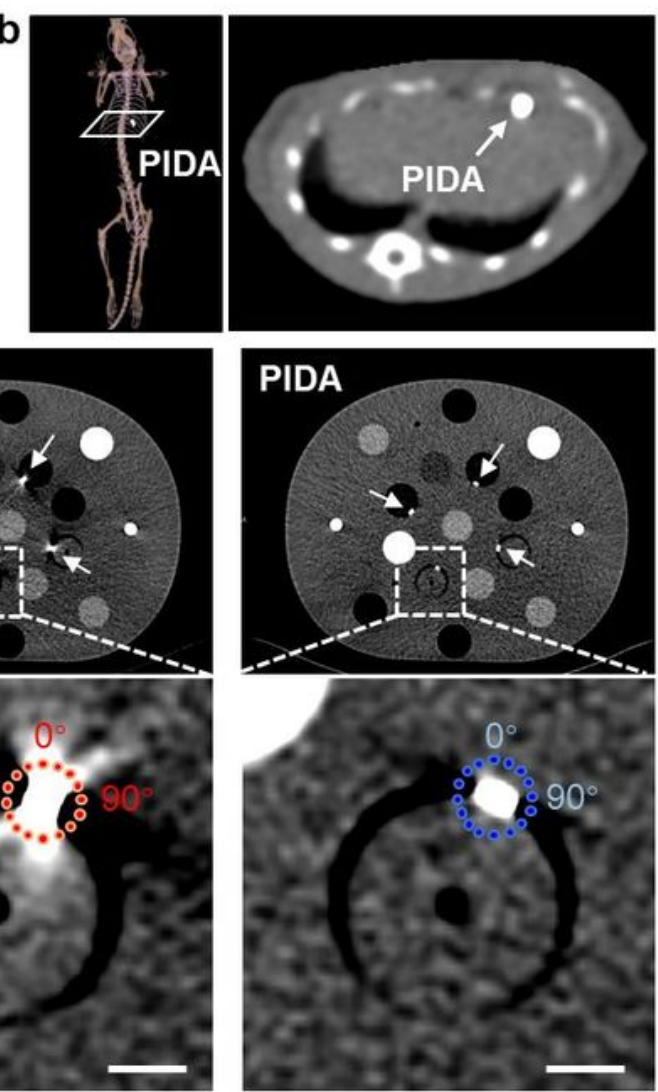

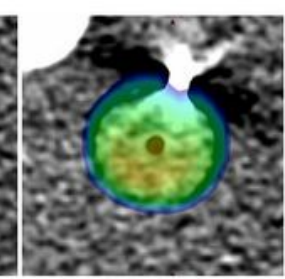

$\mathrm{Au}$

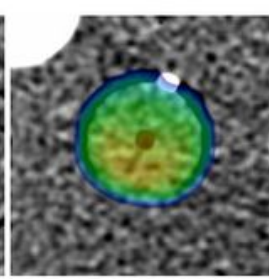

PIDA

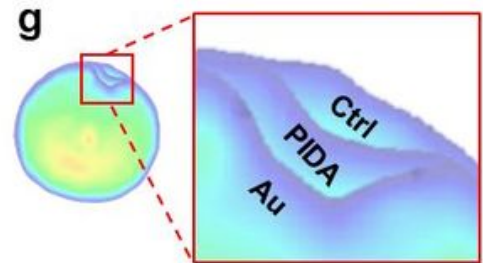

Merge

Figure 5

Accurate radiotherapy dose distribution planning based on PIDA fiducial marker. a CT images of the rat implanted with an Au fiducial marker. b CT images of the rat implanted with a PIDA fiducial marker. $\mathbf{c}$ CT images of thorax phantoms with movable plugs. Scale bar: $1 \mathrm{~cm}$. Control: Thorax phantom with no marker inserted; Au: Thorax phantom with 4 Au markers inserted (pointed by white arrows); PIDA: Thorax phantom with 4 PIDA markers inserted (pointed by white arrows). d,e Quantified relative CT intensities of 
circled spot arrays in $\mathbf{c}$. f Radiation dose distribution for RRT planning calculated on basis of the CT values of the corresponding thorax phantoms in $\mathbf{c}$. $\mathbf{g}$ Overlapping of the calculated radiation dose distribution of Control (Ctrl), Au, and PIDA.
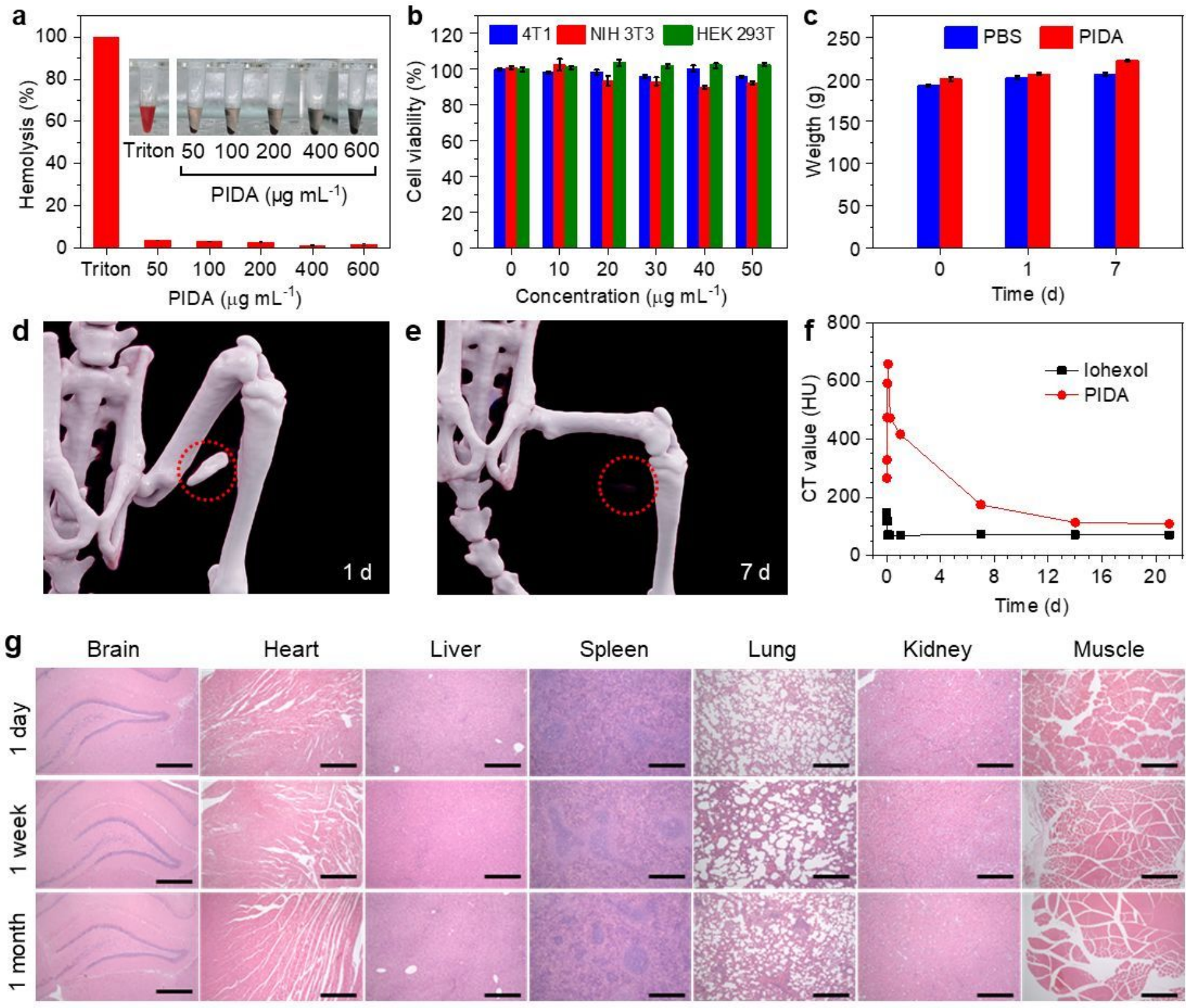

Figure 6

Biosafety assessment of PIDA. a Hemolysis assay and images (insert) of RBCs incubated with different samples. The percentage of RBC lysis was $<5 \%$ for PIDA. b Viability of $4 T 1$, NIH 3T3, and HEK 293T cells pretreated with a series of doses of PIDA. c Plots of CT values of PIDA and iohexol ([I]: $4 \mathrm{mg} \mathrm{mL}^{-1}, 200$ 
$\mu \mathrm{L}$ ) injected in rats as a function time. $\mathbf{d , e}$ CT images of PIDA degradation in muscle/tumor. $\mathbf{f}$ Body weights of rats received the treatment and control rats $(n=3) . \mathbf{g ~ H} \&$ E staining of main organs after being injected with PIDA. Scale bars: $0.5 \mathrm{~mm}$.

\section{Supplementary Files}

This is a list of supplementary files associated with this preprint. Click to download.

- PIDACTSI20220206.docx 